\title{
Commentary
}

\section{On the current neuroenhancement use of transcranial direct current stimulation by healthy individuals-a non-fictional snap-shot: commentary on Lapenta et al. 2014}

\author{
Elisabeth Hildt \\ Johannes Gutenberg-Universität Mainz, Mainz, Rhineland-Palatinate, Germany
}

\begin{abstract}
This commentary examines the pros and cons of the fictitious enhancement scenarios used in Lapenta et al. 2014. Then it gives a non-fictional impression of the current self-enhancement use of transcranial direct current stimulation (tDCS) by healthy individuals and discusses the ethical issues involved.
\end{abstract}

Received 21 January 2014; accepted 23 January 2014. Available online 27 June 2014.

The contribution by Lapenta et al. raises important ethical questions on the use of transcranial direct current stimulation (tDCS) by healthy individuals for enhancement purposes that undoubtedly need further interdisciplinary discussion.

The authors describe fictitious enhancement scenarios each followed by a detailed description of the current tDCS research and by a reflection on the ethical issues involved. The case studies are intuitively appealing and may serve to prompt and facilitate the discussion of the complex ethical issues involved in the enhancement use of non-invasive brain stimulation in general and tDCS in particular. I agree with the authors that the application of narratives and vignettes may be an interesting and emotionally engaging starting point for the discussion of complex ethical issues in the field of medicine.

Having said this, I would like to stress that the fictitious scenarios bear a considerable risk. Being intuitively appealing, the way the fictitious case studies are presented makes them to be kept in mind very easily. Whereas the discussion of facts that follows each of the scenarios is very down-to-earth and reviews the recent empirical research, which shows that currently there are if at all only limited enhancement effects of tDCS in very specific research settings, the fictitious scenarios that stick in mind suggest something different. In most of the scenarios mentioned, non-invasive brain stimulation perfectly works to enhance cognition or to

Elisabeth Hildt, Department of Philosophy, Johannes Gutenberg-Universität, Mainz, Rhineland-Palatinate, Germany. Correspondence regarding this article should be directed to: Elisabeth Hildt, Philosophisches Seminar, Johannes Gutenberg-Universität Mainz, Jakob Welder-Weg 18, 55099 Mainz. Phone: +49 (0) 6131/39-27402. E-mail: hildt@uni-mainz.de modify personality in healthy individuals and seems to be a success in the eyes of those who want the technology administered. Even the last scenario in which safety is highlighted as a central theme ends up positively in the sense that no negative long term effects are observed. However, it is important to emphasize that up to now, in reality, none of these enhancement effects can be achieved by tDCS. Thus, the scenarios may prove to be misleading as they give the inadequate impression that things are much easier and much more effective than they are in reality. This fosters some sort of "what-if-all-practicaland-medical-problems-were-solved" debate in which part of the actual key issues such as safety or efficacy are not adequately taken into consideration any more.

It is plausible to assume that advances in research might in the not-so-distant future lead to possible enhancement uses of tDCS technology. A recent example that may hint at possible future neuroenhancement approaches is a study showing that tDCS applied during a nap significantly improved subsequent encoding of information (Antonenko et al. 2013).

The discrepancy between fictitious scenarios and reality leads me to discuss in more detail an application context with significant ethical implications that is of central relevance for current and possible future neuroenhancement uses of tDCS: self-enhancement. In contrast to the fictitious scenarios described in the article by Lapenta et al., it seems that tDCS for enhancement purposes will rarely be administered by medical doctors such as the fictitious Dr. Icarus or by other medical professionals. Instead, at least currently, the most realistic neuroenhancement scenario is self-administration of tDCS, a form of do-it-yourself brain stimulation.

Right now, there are already companies offering tDCS headsets or tDCS transcranial stimulation kits 
starting from around US\$ 250 (cf.: http://www.foc. us/; http:/www.trans-cranial.com/). In addition, users cultivate a vivid online exchange of experiences with self-administered tDCS as well as instructions for doit-yourself setup and use (cf.: http:/wwwdiytcds.com; http://www.reddit.com/r/tDCS/; http://speakwisdom. wordpress.com/; http://neurogadget.com/2013/12/02/ foc-us-headset-trial-run/9048). The fact that tDCS only needs a portable system which in principle can be applied easily, be it commercially sold or do-it-yourself made, makes tDCS a technique that can be used by lay persons in home experiments.

However, the self-application of tDCS is fraught with considerable problems (Hamilton et al. 2011; Fitz \& Reiner 2013). Whereas in the literature it has been stated that tDCS is apparently safe or relatively safe as well as relatively efficient (Cohen Kadosh et al. 2012; Fitz \& Reiner 2013; Levasseur-Moreau et al. 2013), it is important to stress that up to now not much is known concerning the effects of tDCS on healthy individuals (Hamilton et al. 2011; Cohen Kadosh et al. 2012; Levasseur-Moreau et al. 2013; Sehm \& Ragert 2013). It may be the case that through tDCS an ability is enhanced, but at the cost of it being detrimental to another ability, which may result in mixed effects (Iuculano \& Cohen Kadosh 2013). In addition, there may be unforeseen side-effects and risks. Particularly, the consequences of repeated administration of tDCS on healthy individuals over a longer time span are far from clear (Levasseur-Moreau et al. 2013; Sehm \& Ragert 2013). Furthermore, up to now, the effects of tDCS in real-life situations are not known (Levasseur-Moreau et al. 2013; Sehm \& Ragert 2013). It is not at all farfetched to assume that modifications in cognitive skills, mood or social cognition may have various implications in individual and social life.

When a procedure is offered in a medical setting, the patient or user can expect a medical professional with expert knowledge to adhere to fundamental medical standards concerning safety, risk-benefit-balancing, informed consent, and related aspects. In do-it-yourself administration, however, none of this can be expected. In addition, a regulatory framework for self-administered tDCS does not yet exist (Fitz \& Reiner 2013).

You may say that in self-enhancement, it is up to the individual to deliberately choose to take the risks involved. However, as reliable information on the risks and benefits and on appropriate stimulation parameters is more than scarce, truly informed decision- making is almost impossible at the moment. Individuals may naively underestimate the risks and overestimate the putative benefits. The self-enrolled human trials result in anecdotal stories providing subjective reports of individual experiences and putative enhancement effects. Overall, it seems that technophile individuals experiment with tDCS just for fun, seeking thrill when experimenting with their brains. This rather careless conduct seems to be based on the potentially erroneous intuition that there are no risks involved as tDCS is a non-invasive procedure.

In view of this situation, it is important to inform the public on the current lack of knowledge in this field. Empirical research has to be run in order to provide relevant empirical data on the short- and long-term consequences of tDCS on healthy individuals and on possible tDCS enhancement effects. In addition, detailed knowledge of the current practice and prevalence of do-it-yourself tDCS is needed. Hopefully, a better understanding of the situation will form the basis for adequate regulations providing some guidance for commercial and noncommercial contexts in a field that undoubtedly will be difficult to regulate (cf. Fitz \& Reiner 2013).

\section{References}

Antonenko, D., Diekelmann, S., Olsen, C., Born, J., \& Mölle, M. (2013). Napping to renew learning capacity: enhanced encoding after stimulation of sleep slow oscillations. European Journal of Neuroscience, 37(7), 1142-1151

Cohen Kadosh, R., Levy, N., O’Shea, J., Shea, N., \& Savulescu, J. (2012). The neuroethics of non-invasive brain stimulation. Current Biology, 22(4), R108-R111.

Fitz, N. S., \& Reiner, P. B. (Online First, June 3, 2013). The challenge of crafting policy for do-it-yourself brain stimulation. Journal of Medical Ethics. DOI: 10.1136/medethics-2013-101458.

Hamilton, R., Messing, S., \& Chatterjee, A. (2011). Rethinking the thinking cap: Ethics of neural enhancement using noninvasive brain stimulation. Neurology, 76, 187-193.

Iuculano, T., \& Cohen Kadosh, R. (2013). The mental cost of cognitive enhancement. Journal of Neuroscience, 33(10), 4482-4486.

Lapenta, O., Valasek, C., Brunoni, A., \& Boggio, P. (2014). An ethical discussion of the use of transcranial direct current stimulation for cognitive enhancement in healthy individuals: a fictional case study. Psychology \& Neuroscience. DOI: 10.3922/j.psns.2014.02.03

Levasseur-Moreau, J., Brunelin, J., \& Fecteau, S. (2013). Noninvasive brain stimulation can induce paradoxical facilitation. Are these neuroenhancements transferable and meaningful to security services? Frontiers in Human Neuroscience, 7, 449. DOI: 10.3389/ fnhum.2013.00449.

Sehm, B., \& Ragert, P. (2013). Why non-invasive brain stimulation should not be used in military and security services. Frontiers in Human Neuroscience, 7, 553. DOI: 10.3389/fnhum.2013.00553. 